\title{
Biallelic GALM pathogenic variants cause a novel type of galactosemia
}

\author{
Yoichi Wada, MD¹, Atsuo Kikuchi, MD, PhD ${ }^{1}$, Natsuko Arai-Ichinoi, MD, PhD ${ }^{1}$, \\ Osamu Sakamoto, MD, PhD ${ }^{1}$, Yusuke Takezawa, MD ${ }^{1}$, Shinya Iwasawa, MD ${ }^{1}$, \\ Tetsuya Niihori, MD, PhD², Hiromi Nyuzuki, MD³, Yoko Nakajima, MD, PhD ${ }^{4}$, Erika Ogawa, MD, PhD ${ }^{5}$, \\ Mika Ishige, MD, PhD ${ }^{5}$, Hiroki Hirai, MD, PhD ${ }^{6}$, Hideo Sasai, MD, PhD, Ryoji Fujiki, $\mathrm{MS}^{8}$, \\ Matsuyuki Shirota, MD, $\mathrm{PhD}^{9}$, Ryo Funayama, $\mathrm{PhD}^{10}$, Masayuki Yamamoto, MD, $\mathrm{PhD}^{11}$, \\ Tetsuya Ito, MD, $\mathrm{PhD}^{4}$, Osamu Ohara, $\mathrm{PhD}^{8}$, Keiko Nakayama, MD, PhD ${ }^{10}$, Yoko Aoki, MD, PhD ${ }^{2}$, \\ Seizo Koshiba, $\mathrm{PhD}^{11}$, Toshiyuki Fukao, $\mathrm{MD}, \mathrm{PhD}^{7}$ and Shigeo Kure, MD, $\mathrm{PhD}^{1,11}$
}

\begin{abstract}
Purpose: Galactosemia is caused by metabolic disturbances at various stages of galactose metabolism, including deficiencies in enzymes involved in the Leloir pathway (GALT, GALK1, and GALE). Nevertheless, the etiology of galactosemia has not been identified in a subset of patients. This study aimed to explore the causes of unexplained galactosemia.

Methods: Trio-based exome sequencing and/or Sanger sequencing was performed in eight patients with unexplained congenital galactosemia. In vitro enzymatic assays and immunoblot assays were performed to confirm the pathogenicity of the variants.

Results: The highest blood galactose levels observed in each patient were $17.3-41.9 \mathrm{mg} / \mathrm{dl}$. Bilateral cataracts were observed in two patients. In all eight patients, we identified biallelic variants (p.Arg82*, p.Ile99Leufs ${ }^{\star} 46$, p.Gly142Arg, p.Arg267Gly, and p.Trp311*) in the GALM encoding galactose mutarotase, which
\end{abstract}

catalyzes epimerization between $\beta$ - and $\alpha$-D-galactose in the first step of the Leloir pathway. GALM enzyme activities were undetectable in lymphoblastoid cell lines established from two patients. Immunoblot analysis showed the absence of the GALM protein in the patients' peripheral blood mononuclear cells. In vitro GALM expression and protein stability assays revealed altered stabilities of the variant GALM proteins.

Conclusion: Biallelic GALM pathogenic variants cause galactosemia, suggesting the existence of type IV galactosemia.

Genetics in Medicine (2018) https://doi.org/10.1038/s41436-018-0340-x

Keywords: galactose; galactose mutarotase; GALM; genetics; Leloir pathway

\section{INTRODUCTION}

Lactose accounts for almost all sugar present in breast milk or formula and is a major source of energy for newborns and infants. Lactose is cleaved into D-glucose and $\beta$-D-galactose on the intestinal villi by $\beta$-D-galactosidase. $\beta$-D-Galactose is taken up into the liver via the portal vein and primarily metabolized by the Leloir pathway, which is highly conserved and consists of four individual enzymes: galactose mutarotase (or aldose 1-epimerase) (GALM, EC 5.1.3.3), galactokinase (GALK1, EC 2.7.1.6), galactose-1-phosphate uridylyltransferase (GALT, EC 2.7.7.12), and UDP-galactose-4'-epimerase
(GALE, EC 5.1.3.2) (Supplementary Figure S1) (refs. ${ }^{1-3}$ ). Galactosemia is one of the disorders detected in newborn screens ${ }^{4}$ and is caused by disturbances in various steps of galactose metabolism, including deficiencies in the enzymes in the Leloir pathway, portosystemic shunts, cholestasis (e.g., citrin deficiency [MIM 605814] [refs. ${ }^{5,6}$ ] and neonatal hepatitis), and other metabolic disorders, such as Fanconi-Bickel syndrome (MIM 227810). The first step of the Leloir pathway involves epimerization between $\beta$ - and $\alpha$ D-galactose, which is catalyzed by GALM. ${ }^{7}$ The next three genes in the Leloir pathway (GALT [MIM 606999] [refs. ${ }^{8,9}$ ],

\footnotetext{
${ }^{1}$ Department of Pediatrics, Tohoku University School of Medicine, Sendai, Japan; ${ }^{2}$ Department of Medical Genetics, Tohoku University School of Medicine, Sendai, Japan; ${ }^{3}$ Department of Pediatrics, Niigata University School of Medicine, Niigata, Japan; ${ }^{4}$ Department of Pediatrics, Fujita Health University School of Medicine, Toyoake, Japan; ${ }^{5}$ Department of Pediatrics and Child Health, Nihon University School of Medicine, Tokyo, Japan; ${ }^{6}$ Department of Pediatrics, Ehime Prefectural Central Hospital, Matsuyama, Japan; ${ }^{7}$ Department of Pediatrics, Graduate School of Medicine, Gifu University, Gifu, Japan; ${ }^{8}$ Department of Technology Development, Kazusa DNA Research Institute, Kisarazu, Japan; ${ }^{9}$ Division of Interdisciplinary Medical Sciences, United Centers for Advanced Research and Translational Medicine, Tohoku University Graduate School of Medicine, Sendai, Japan; ${ }^{10}$ Division of Cell Proliferation, United Centers for Advanced Research and Translational Medicine, Tohoku University Graduate School of Medicine, Sendai, Japan; ${ }^{11}$ Tohoku Medical Megabank Organization, Tohoku University, Sendai, Japan. Correspondence: Atsuo Kikuchi (akikuchi-thk@umin.ac.jp)

These authors contributed equally: Yoichi Wada, Atsuo Kikuchi, Natsuko Arai-Ichinoi and Osamu Sakamoto
} 
GALK1 [MIM 604313] [ref. ${ }^{10}$ ], and GALE [MIM 606953] [refs. ${ }^{11-13}$ ]) have been identified as being responsible for type I, II, and III galactosemia (GALT [MIM 230400], GALK1 [MIM 230200], and GALE [MIM 230350] deficiencies), respectively. ${ }^{1,7}$

In patients with galactosemia, appropriate care and precise diagnosis are essential to avoid complications. Unless breast milk or normal formula is substituted with galactose/lactosefree formula, cataracts often develop. Severe symptoms, including a failure to thrive, gastrointestinal symptoms, liver dysfunction, sepsis, and death, may occur in patients with classic galactosemia (severe GALT deficiency) ${ }^{2}$ or patients with a generalized GALE deficiency. ${ }^{14,15}$ In adolescent and adult patients with a GALT deficiency, long-term complications, such as neuropsychological symptoms and premature ovarian failure, have also been observed. ${ }^{16}$ Surgical intervention may be required in certain patients with portosystemic shunts. Thus, a differential diagnosis of the type of galactosemia is crucial for appropriate care. Nevertheless, the etiology of some galactosemia cases remains unknown. ${ }^{17}$ We encountered a group of patients with unexplained and persistent galactosemia who had did not present with pathogenic variants in GALT, GALK1, or GALE, portosystemic shunts, cholestasis, or no urinary glucose, which is commonly present in patients with Fanconi-Bickel syndrome.

We performed exome sequencing in these patients with unexplained galactosemia to explore the cause of this condition, and identified biallelic pathogenic variants in GALM (MIM 137030) for the first time. We describe the genetic and clinical features of a novel type of galactosemia associated with GALM deficiency in the present study.

\section{Patients}

\section{MATERIALS AND METHODS}

We studied eight Japanese patients with unexplained congenital galactosemia (Table 1, Supplemental Note). Initially, we studied two unrelated patients with galactosemia (patients 1 and 2) whose clinical data were not consistent with GALT, GALK1, and GALE deficiencies, portosystemic shunt, cholestatic diseases, or Fanconi-Bickel syndrome. Urine glucose test results were negative in these two patients, excluding a diagnosis of Fanconi-Bickel syndrome. The Beutler test was negative, excluding the possibility of a GALT deficiency. Detection of blood galactose-1-phosphate (Gal-1P) (cut-off value: $15 \mathrm{mg} / \mathrm{dl}$ ) and elevated galactose levels (cutoff value: $3 \mathrm{mg} / \mathrm{dl}$ ) in the two patients in the newborn screen suggested the possibility of a GALE deficiency rather than a GALK1 deficiency; however, their GALE activities in red blood cells were normal (Table 1). Variants in GALT, GALK1, and GALE were screened in the two patients, but no pathogenic variants were identified.

Six additional patients with unexplained congenital galactosemia were enrolled. Their clinical data were not consistent with GALT, GALK1, or GALE deficiencies, or Fanconi-Bickel syndrome (Table 1). Patients 5 and 8 received a diagnosis of galactosemia caused by a portosystemic shunt, but their galactosemia persisted after spontaneous closure of the shunts. No pathogenic variants in GALT, GALK1, and GALE were identified in these six patients, with the exception of a heterozygous variant, c. $446 \mathrm{C}>\mathrm{T}, \mathrm{p}$.(Thr149Met), in GALK1 identified in patient 3 . Although this variant was rare and predicted to affect protein function by certain prediction tools, the amino acid sequence is not conserved among vertebrates. In particular, rhesus monkeys express a methionine, the same residue as the variant allele of patient 3 , in this position. In addition, we did not identify other candidate variants in the same gene. Therefore, we did not pursue this variant further.

The present study was approved by the ethics committee of Tohoku University School of Medicine. Written informed consent was obtained from the patients' parents.

\section{Sequencing analysis}

For patients 1 and 2, we performed trio-based exome sequencing. Genomic DNA was captured using SureSelectXT Human All Exon V6 (60 Mb) Kits (Agilent Technologies, Santa Clara, CA, USA) and sequenced on a HiSeq2500 (Illumina, San Diego, CA, USA) with 126-base pair pairedend reads. The reads were mapped to the hg19 human reference genome using Novoalign v. 2.08 .05 (http://www. novocraft.com), and single-nucleotide variants (SNVs) and insertions and/or deletions (indels) were identified using the Genome Analysis Toolkit (GATK) v. 1.6-13 (ref. ${ }^{18}$ ). After quality filtering steps, variants were annotated using ANNOVAR. $^{19}$ Nonsynonymous SNVs, splice-site variants, and indels were retained. Because both sexes were affected, we hypothesized an autosomal recessive mode of inheritance. We excluded variants present at $<1 \%$ minor allele frequency (MAF) in the 1000 Genomes database, the ExAC database version 0.3 (all and East Asian populations), and the Human Genetic Variation Database (HGVD) ${ }^{20,21}$ For patients 3-8, Sanger sequencing of all exons and flanking regions of GALM was performed.

\section{In vitro expression analysis}

The GALM complementary DNA (cDNA) (IRAK015M11) was provided by the RIKEN Bioresource Center (BRC) through the National Bio-Resource Project of the Ministry of Education, Culture, Sports, Science and Technology (MEXT), Japan, ${ }^{22-25}$ and was subcloned into pCMV6-AN-DDK (PS100014, OriGene, Rockville, MD, USA) or pET21d(+) (69743, Novagen, Merck Millipore, Darmstadt, Germany) with a His6 tag at the N-terminus using the In-Fusion HD Cloning Kit (TaKaRa, Shiga, Japan). Then, 293FT cells (R70007, Invitrogen $\left.{ }^{\text {Tx}}\right)$ were cultured in Dulbecco's modified Eagle's medium (DMEM) containing high glucose supplemented with $10 \%$ fetal bovine serum, and $1 \%$ penicillin and streptomycin $\left(100 \mathrm{U} / \mathrm{ml}\right.$ and $100 \mu \mathrm{g} / \mathrm{ml}$, respectively) at $37^{\circ} \mathrm{C}$ in a $5 \% \mathrm{CO}_{2}$ incubator. Epstein-Barr virus transformed lymphoblastoid cell lines (EBV-LCLs) were established from patients 2, 3, 8, and healthy controls using previously described methods. ${ }^{26}$ All cell lines were tested for mycoplasma 


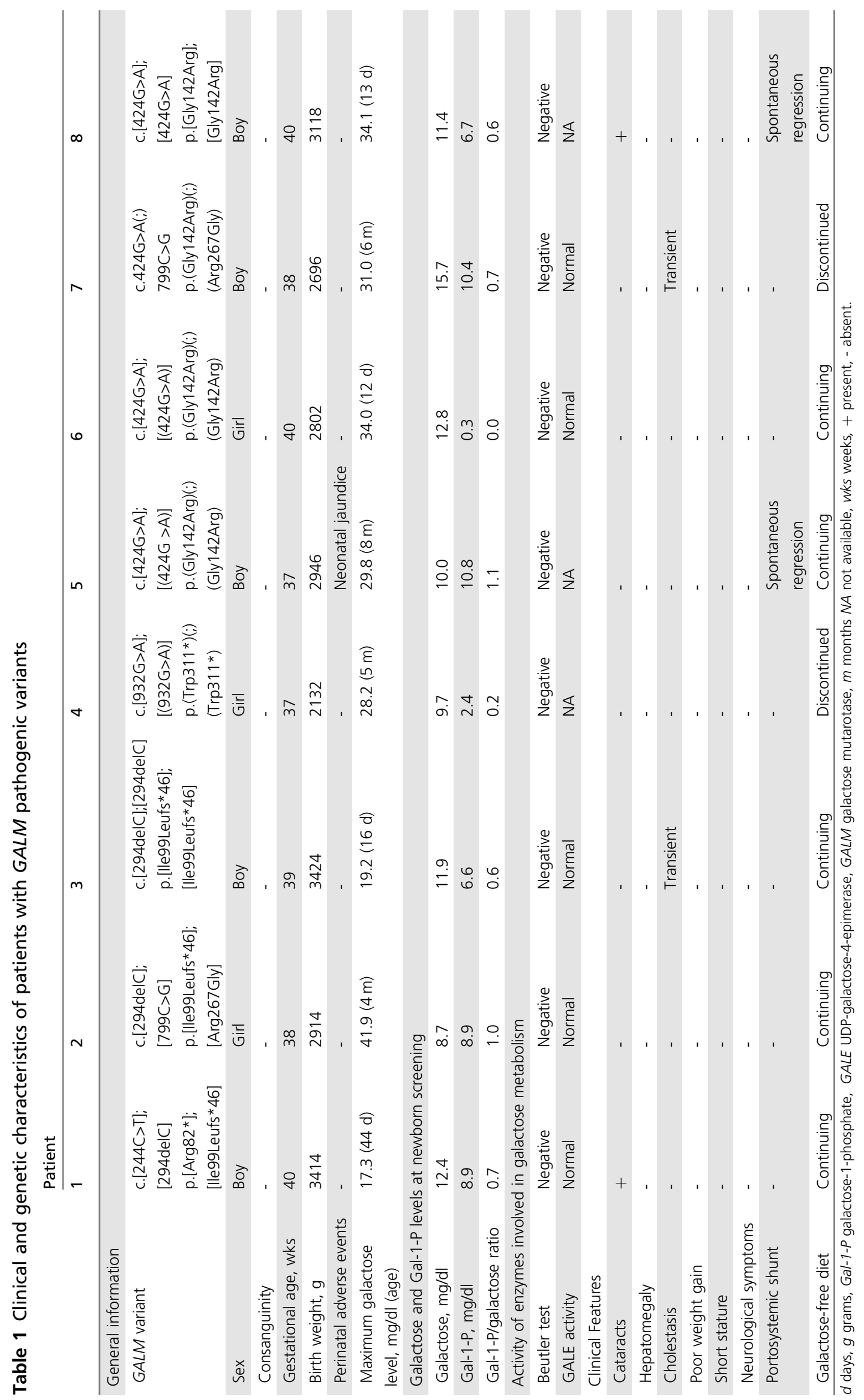


contamination using a MycoAlert ${ }^{\mathrm{Tm}}$ Mycoplasma Detection Kit (LT07-118, Lonza, Basel, Switzerland).

\section{Cycloheximide protein stability assay}

The pCMV6-FLAG-GALM (wild-type, G142R, R267G, W311*) plasmid was transfected into 293FT cells using Lipofectamine LTX (15338100, Thermo Fisher Scientific Inc., MA). Twenty-four hours after transfection, cells were treated with $20 \mu \mathrm{g} / \mathrm{ml}$ of cycloheximide. Lysates were harvested 0,1 , 4 , or 12 hours after treatment and subjected to an immunoblot analysis, as described below.

\section{Immunoblot analysis}

We performed immunoblot assays on proteins extracted from peripheral blood mononuclear cells (PBMCs) or 293FT cells expressing FLAG-GALM. Antibodies used in the present study were anti-FLAG (F3165, Sigma-Aldrich, Merck Millipore), anti-GALM (16022-1-AP, Proteintech, Rosemont, IL, USA), peroxidase-conjugated anti- $\beta$-Actin (017-24573, Wako, Osaka, Japan), HRP-conjugated anti-mouse IgG (NA9310V, GE Healthcare UK Ltd, Buckinghamshire, England), and HRP-conjugated antirabbit IgG (NA9340V, GE Healthcare UK Ltd).

\section{Analysis of GALM enzyme activity in EBV-LCLs}

GALM enzyme activity was measured in EBV-LCLs obtained from the patients using a previously described coupled assay, $7,27,28$ with minor modifications. GALM catalyzes the conversion of $\alpha$-D-galactose to $\beta$-D-galactose, followed by the reduction of $\mathrm{NAD}^{+}$to $\mathrm{NADH}$ coupled with the reaction of $\beta-\mathrm{D}$ galactose to $\beta$-galactonic acid catalyzed by $\beta$-galactose dehydrogenase. This reaction was performed in a solution containing 360 $\mu \mathrm{M}$ freshly dissolved $\mathrm{D}(+)$-galactose (071-00032, Wako); $3 \mathrm{mM}$ $\mathrm{NAD}^{+} ; 2.5 \mathrm{U} \beta$-galactose dehydrogenase; $10 \mathrm{mM}$ HEPES, $\mathrm{pH}$ 7.4; and approximately $14 \mu \mathrm{g}$ of EBV-LCL lysate. We confirmed that the $\mathrm{D}(+)$-galactose almost completely consisted of $\alpha$-D-galactose using nuclear magnetic resonance (NMR) (Supplementary Figure S2). The rate of increase in the absorbance of NADH at $339 \mathrm{~nm}$ was monitored using a SPECTRA MAX 190 instrument (Molecular Devices, CA, USA) immediately after the dissolution of $\alpha$-D-galactose. These rates were corrected by subtracting the absorbance measured in the absence of cell lysates. We were not able to measure the GALM activity using PBMCs because control PBMCs exhibit very low enzymatic activity.

\section{Statistical analysis}

The statistical analysis was performed with JMP Pro 13.1.0. $P$ values $<0.05$ were considered statistically significant.

\section{RESULTS}

\section{Identification of pathogenic variants in GALM and the spectrum of pathogenic variants}

Initially, we performed trio-based exome sequencing on two unrelated patients (patients 1 and 2). The mean depth of each exome was approximately 100. Trio-based exome sequencing revealed only one candidate gene, GALM (NM_138801.2), for an autosomal recessive model (Supplementary Table S1). No rare de novo variants shared by the two probands were identified. An additional Sanger sequencing analysis identified six patients with unexplained congenital galactosemia and biallelic variants in GALM. Three patients (patients 1, 3, and 4) were homozygous or compound heterozygous for stop-gain variants or a frameshift variant leading to a premature termination in GALM; three patients (patients 5, 6, and 8) were homozygous for a missense variant, p.Gly142Arg; one patient (patient 7) was compound heterozygous for different missense variants; and one patient (patient 2) was compound heterozygous for missense and nonsense GALM variants (Table 1). Collectively, we identified five different GALM pathogenic variants (three stop-gain and two missense) in eight patients from unrelated families of Japanese descent (Table 1, Supplementary Table S2). All variants identified in the GALM gene are rare $(<0.1 \% \mathrm{MAF})$, and no homozygous carriers of these variants were identified among 60,706 exomes in ExAC or 1208 people of Japanese ancestry in HGVD, whereas only one homozygous carrier of c.932G $>A$, p.Gly142Arg from Africa, was identified in gnomAD (Supplementary Table S2). Moreover, no homozygous stopgain or frameshift genotypes in GALM were found in these databases. Approximately half $(7 / 16)$ of the variants were $\mathrm{p}$. Gly142Arg. According to the multiple sequence alignment, two missense variants, p.Gly142Arg and p.Arg267Gly, affect evolutionarily conserved residues (Supplementary Figure S3). The two missense variants were also predicted to be pathogenic by different in silico prediction tools (SIFT, PolyPhen-2, and MutationTaster) (Supplementary Table S2).

\section{Measurement of GALM enzyme activity in EBV-LCLs using a coupled assay}

We measured the mutarotase activity using a coupled assay. EBV-LCLs were available from two patients (patients 2 and 3). Patients' EBV-LCLs were unable to convert $\alpha$-D-galactose to $\beta$-D-galactose, but the rate of $\alpha$-D-galactose conversion in three control samples was greater than a buffer control (Fig. 1).

\section{The GALM protein was not expressed in patients' PBMCs}

PBMCs were available from three patients (patients 2, 3, and 8) carrying biallelic GALM variants. An immunoblot analysis with an anti-GALM antibody revealed the lack of the GALM protein in PBMCs from all three patients compared with control cells (Fig. 2a). Among the three patients, patients 2 and 8 harbored two missense variants and stop-gain or frameshift variants in GALM (p.[Ile99Leufs`46];[Arg267Gly] and p.[Gly142Arg]; [Gly142Arg]), respectively. Based on this result, the stability of the variant GALM proteins is affected in cells harboring the missense variants.

\section{Exogenous variant GALM expression was reduced in 293FT cells}

We overexpressed the wild-type or variant FLAG-tagged GALM protein in 293FT cells to evaluate the possibility that 


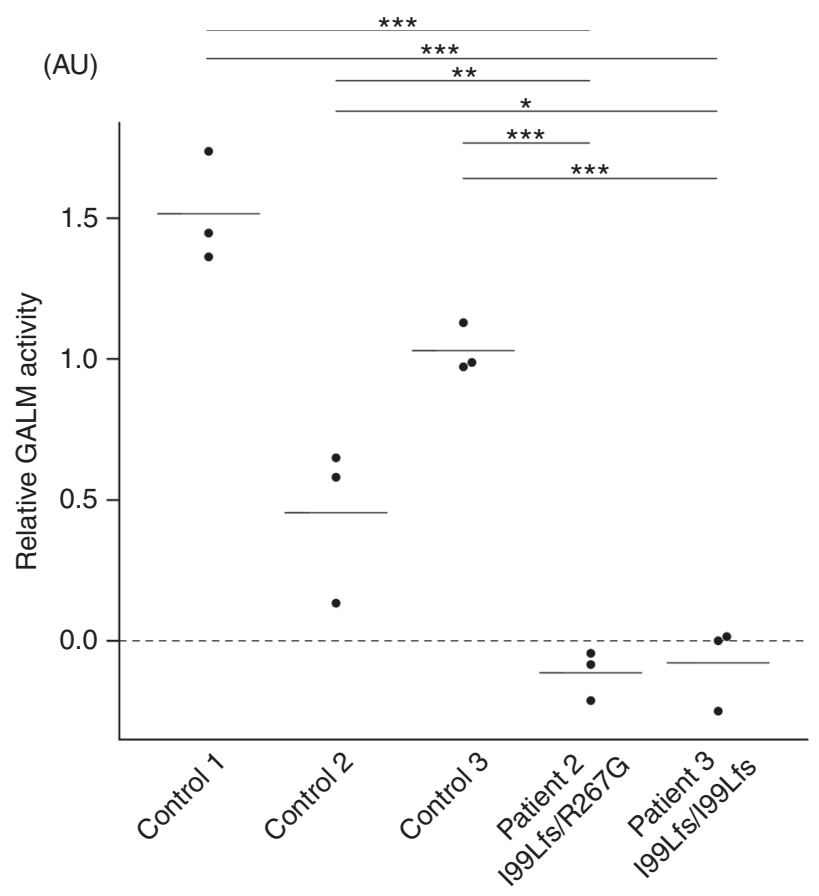

Fig. 1 Relative GALM enzyme activities in peripheral blood mononuclear cells (PBMCs), as determined using coupled assays. Individual data and means of three independent experiments are shown. The average of three controls in each experiment was set to 1 . $A U$ arbitrary units. ${ }^{*} P=$ $0.03,{ }^{*} P=0.02, * * * P<0.001$ (Tukey-Kramer test).

the pathogenic variants identified in patients affect the stability of the GALM protein. Among the variants, R82* and I99Lfs variants produced no or low levels of truncated protein, and other variants, G142R, R267G, and W311*, showed compromised expression of GALM (Fig. 2b).

\section{Pathogenic variants in GALM affect the stability of GALM proteins}

The compromised expression of the three variants prompted us to evaluate whether these variant GALM proteins exhibit a shorter half-life. According to the results of the cycloheximide protein stability assays, G142R, R267G, and W311* variants markedly reduced protein stability, as the variant proteins displayed shorter half-lives than the wild-type protein (Fig. 2c).

Recombinant variant GALM proteins yielded approximately $\mathbf{5 0 \%}$ less protein upon expression in $E$. coli than the wildtype protein

We prepared recombinant GALM proteins to determine the specific activity of the GALM missense variants. Although the yield of the wild-type protein was approximately $5 \mathrm{mg}$ of GALM protein per liter of bacterial culture, the respective yields of the two missense constructs (G142R, R267G) were approximately $1 \%$ and $4 \%$ of the yield of the wild-type GALM protein. This reduction occurred because most of the variant proteins were present in the insoluble fraction (data not shown), suggesting that the two missense pathogenic variants affect protein folding. The enzymatic activity of the recombinant proteins carrying the two missense variants was approximately half of the activity of the wild-type protein (Supplementary Figure S4). This outcome is in line with the fact that the two residues (G142 and R267) are far from the active site that is involved in sugar binding ${ }^{29}$ (Supplementary Figure S5).Thus, the two missense variants affect the quantity, but not the quality, of GALM proteins, consistent with the results of the immunoblot analysis of PBMCs.

\section{The phenotypic spectrum of patients carrying GALM pathogenic variants}

The highest blood galactose levels observed in each patient carrying GALM pathogenic variants were $17.3-41.9 \mathrm{mg} / \mathrm{dl}$ (Table 1, Supplemental Note). The Gal-1-P levels were highest in the neonatal period and decreased below $1 \mathrm{mg} / \mathrm{dl}$ in early infancy in each patient (Table 1, Supplemental Note, Fig. 3). None of the eight patients presented with gastrointestinal symptoms or severe liver dysfunction, which are caused by a GALT deficiency or generalized GALE deficiency. Two patients (patients 1 and 8) presented with bilateral cataracts. The presence of long-term complications, such as neuropsychological impairments or premature ovarian failure, was not clear because no adult patients were examined in the present study, although we do not have these complications to date. All patients were treated with a galactose-restricted diet immediately after the diagnosis of galactosemia, and therefore the natural history of GALM deficiency remained unknown. Two of these patients (patients 4 and 7) were allowed to resume a normal diet.

By comparing Gal-1-P/galactose ratios in newborn screens obtained from patients with a GALM deficiency, GALE deficiency, and portosystemic shunt using in-house data, we were able to differentiate between GALM deficiency and GALE deficiency. The ratios observed in patients with a GALM deficiency were similar to the ratios observed in patients with a portosystemic shunt (Fig. 4). Two patients (patients 5 and 8) carrying GALM pathogenic variants also had portosystemic shunts.

\section{DISCUSSION}

We identified biallelic pathogenic variants in GALM in eight patients with unexplained galactosemia, suggesting the presence of a novel type of galactosemia, namely, type IV galactosemia. Enzymatic activities of GALM were abolished in EBV-LCLs from patients 2 and 3 (Fig. 1). Immunoreactive GALM protein was undetectable in PBMCs from patients 2, 3, and 8 (Fig. 2a). In vitro GALM expression and protein stability assays revealed alterations in the stability of variant GALM proteins. According to the results of the recombinant protein analysis, the two missense pathogenic variants ( $p$. Gly142Arg and p.Arg267Gly) affect the quantity but not the quality of the protein (Fig. 2, Supplementary Figure S4). Thus, the novel type of galactosemia identified in the present study is caused by loss-of-function variants in GALM, which is the only gene that has never previously been associated with 
a
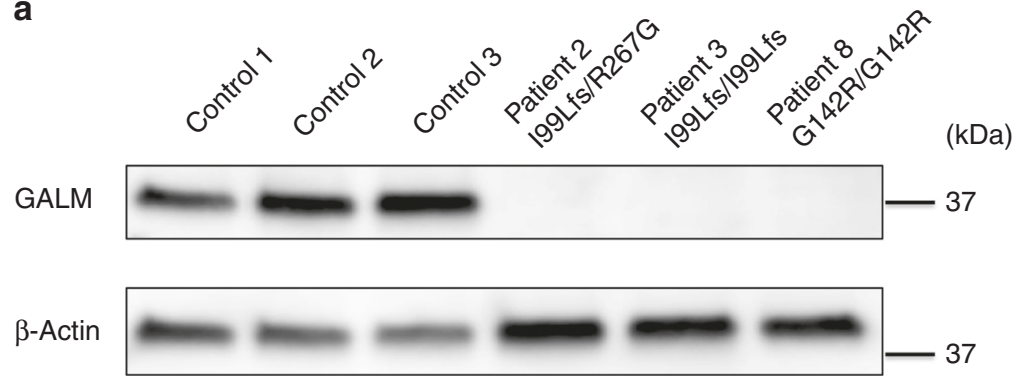

b

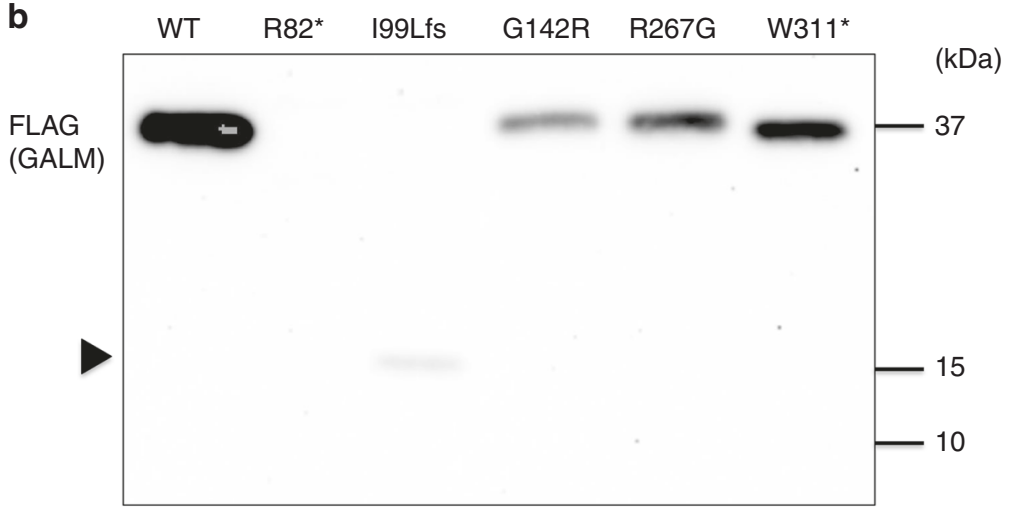

$\beta$-Actin

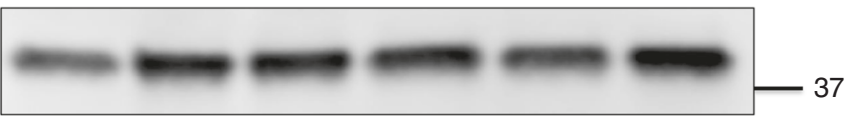

C
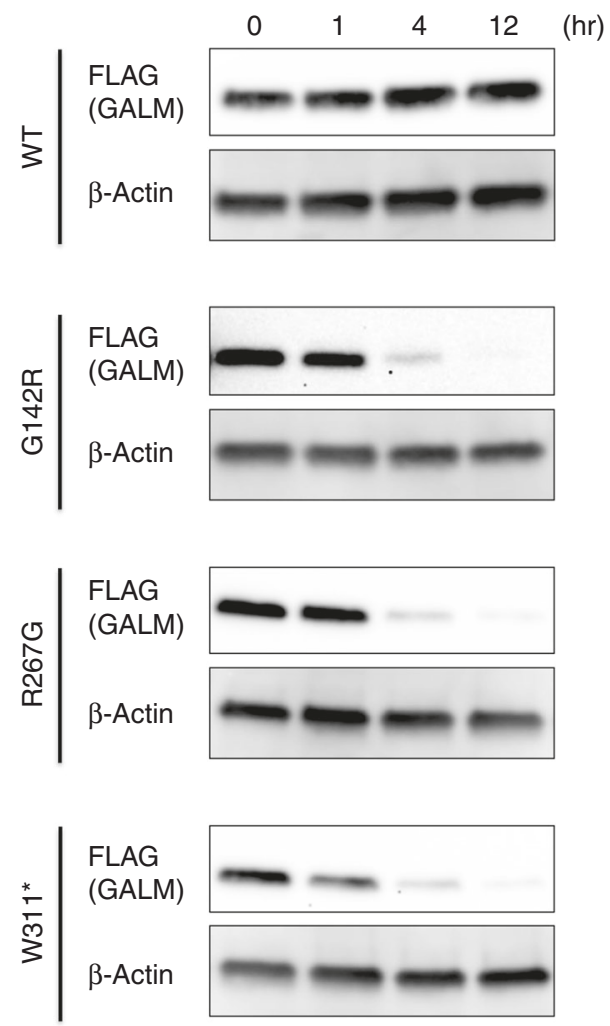

Fig. 2 Alterations in the stability of variant GALM proteins. a GALM expression in peripheral blood mononuclear cells (PBMCs), as determined by immunoblotting. $\beta$-Actin was used as a loading control. Representative results from three independent experiments are shown. $\mathbf{b}$ Immunoblot analysis of GALM expression. Transiently expressed FLAG-GALM was detected with an anti-FLAG antibody. $\beta$-Actin was used as a loading control. The expected position of a truncated protein product stemming from the $199 \mathrm{Lfs}$ allele is indicated with the arrowhead. Representative results from three independent experiments are shown. c Cycloheximide protein stability assay. Times at which cells were harvested after treatment are indicated. $\beta$-Actin was used as a loading control. Representative results from three independent experiments are shown. WT wild type.

galactosemia among the four genes encoding enzymes involved in the Leloir pathway.

GALM deficiency leads to persistent galactosemia, which should be treated appropriately. In this study, when patients with GALM deficiency consumed normal formula or were breast fed transiently before the weaning period, their blood galactose levels were elevated (Table 1, Fig. 3) as high as 41.9 $\mathrm{mg} / \mathrm{dl}$ (Supplemental Note). While nonenzymatic conversion of $\beta$-D-galactose to $\alpha$-D-galactose occurs in water, ${ }^{30}$ galactose mutarotase is necessary for normal cell growth at least in some organisms, including yeast and Escherichia coli, probably because the rate of nonenzymatic conversion is slower in those cells than in water. ${ }^{31,32}$ It is likely that mammals, including humans, similar to yeast and Escherichia coli, also need galactose mutarotase for the efficient anomerization of galactose, particularly during the lactation period, when they consume large quantities of lactose. In addition, bilateral congenital cataracts were observed in two patients (patients 1 and 8 ). In patient 1 , the cataracts were resolved after the start of a galactose-restricted diet (Supplemental Note). This finding indicates that the cataracts were likely caused by galactosemia due to the GALM deficiency. Thus, patients with a GALM deficiency should be diagnosed and treated correctly, although severe complications, such as neurological symptoms, have not developed to date.

The phenotypes of GALM deficiency are similar to those of GALK1 deficiency, which abolishes the next step in the Leloir pathway (Supplementary Figure S1), as the only consistent clinical symptom of GALK1 deficiency is also cataracts. ${ }^{33,34}$ GALM deficiency shows more severe phenotypes than "peripheral" GALE deficiency, which causes virtually no clinical abnormalities except galactosemia. ${ }^{35}$ Because all of the patients enrolled in the present study were treated with a galactose-restricted diet immediately after the diagnosis of galactosemia, the natural history of patients with a GALM deficiency, as well as the long-term prognosis, remain unknown. Although a nonsynonymous polymorphism in GALM has been associated with serotonin transporter binding potential in the human thalamus in a genome-wide association study, ${ }^{36}$ the phenotypic significance of this association remains to be identified. Elucidation of the natural history and long-term prognosis is essential for clinicians to provide an accurate diagnosis of GALM deficiency. 

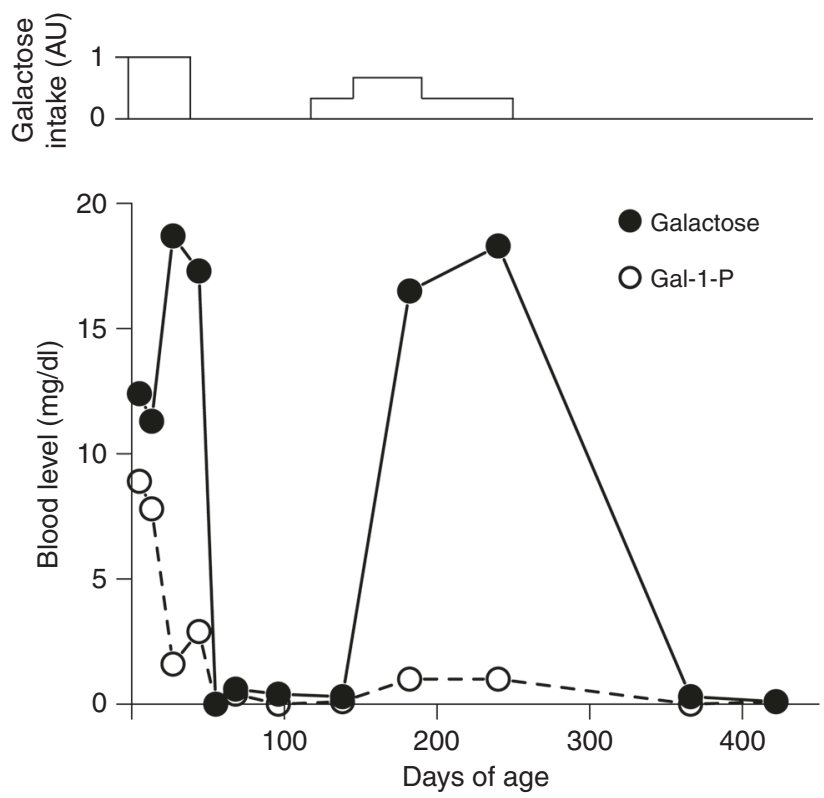

Fig. 3 Time courses of galactose and Gal-1-P levels and the amount of galactose intake in patient 1. The galactose intake is a ratio of the intake of galactose-containing milk (normal formula) to the total milk intake (upper). The blood galactose level was elevated in response to a relaxing of galactose restriction (lower). Gal-1-P galactose-1-phosphate, $A U$ arbitrary units.

Heterozygous carriers of a pathologic GALM variant were identified in public variant databases derived from populations of various ethnicities, suggesting that GALM deficiency exists in many countries. According to gnomAD, a predicted loss-of-function variant, p.Gly212*, was detected in 72 of 25,514 alleles in the Finnish population. Another predicted loss-of-function variant, p.Glu329Argfs ${ }^{\star} 18$, was present in 38 of 33,420 alleles in the Latino population. The p.Gly142Arg variant was identified in 7 of 16 Japanese variant alleles as a loss-of-function variant (Fig. 2). The pathogenic variant was also observed in 152 of 24,026 alleles in the African population. Interestingly, there is one African individual who is homozygous for p.Gly142Arg out of 277,212 alleles in the gnomAD database (Supplementary Table S2). The severity of the disease in homozygotes may be highly variable, particularly in patients with different ethnicities. Particularly in countries that perform a neonatal screen for galactosemia, it is also likely that patients with galactosemia caused by a GALM deficiency received a galactose-restricted diet due to the unexplained galactosemia but were allowed to resume a normal diet after the weaning period without any sequelae such as cataracts, as with our patients 4 and 7 . The restriction for patients with unexplained galactosemia might have prevented us from making the correct diagnosis of GALM deficiency. A carrier frequency analysis would be helpful for evaluating the prevalence of galactosemia caused by GALM deficiency in those countries.

The differentiation between GALM deficiency and portosystemic shunt exclusively based on biochemical and imaging analyses is sometimes difficult. We compared galactose and

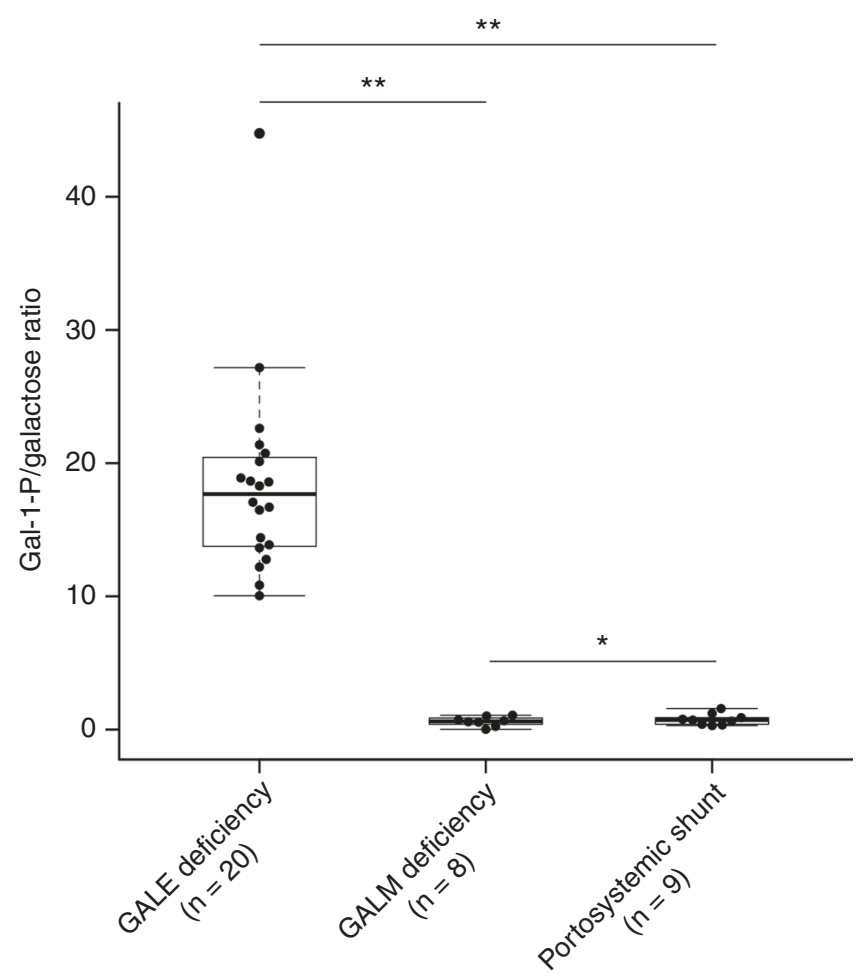

Fig. 4 Comparison of the Gal-1-P/galactose ratios in a newborn screen from patients with a GALM deficiency, GALE deficiency, and portosystemic shunt. In-house data from patients with a GALE deficiency and portosystemic shunts were used. ${ }^{*} P=0.81,{ }^{*} P P<0.001$ (Steel-Dwass test).

Gal-1-P levels in newborn screens between patients with a GALM deficiency, GALE deficiency, and portosystemic shunts. Gal-1-P/galactose ratios in patients with a GALM deficiency were similar to patients with portosystemic shunts, whereas the ratios were significantly elevated in patients with a GALE deficiency (Fig. 4). Notably, patients 5 and 8 were diagnosed with portosystemic shunts based on the presence of high levels of total bile acids and imaging findings at the initial diagnosis of galactosemia. GALM deficiency should be considered when galactosemia persists, despite the disappearance of sonographic findings of a portosystemic shunt or decreased levels of total bile acids. A proposed flow chart for the diagnosis of congenital galactosemia is shown in Supplementary Figure S6.

The mechanism of Gal-1-P fluctuations in patients with GALM deficiency remains to be elucidated. In the neonatal period, the Gal-1-P levels were relatively elevated, while the Gal-1-P levels declined in the postneonatal period (Table 1, Fig. 3, Supplemental Note). The profiles of galactose and Gal-1-P in the postneonatal period are similar to those associated with GALK1 deficiency. A possible explanation for the relatively high Gal-1-P levels in neonatal patients with GALM deficiency is that that galactokinase activities in neonates are higher than those in infants and adults. $^{37}$ Future research should address whether these 
fluctuations in Gal-1-P are unique to patients with GALM deficiency.

The detection of accumulated $\beta$-D-galactose, the substrate of GALM, might enable clinicians to biochemically diagnose GALM deficiency, in addition to a genetic diagnosis. We have established separate detection systems for $\alpha$ - and $\beta$-Dgalactose using NMR (Supplementary Figure S2), and examined the anomers of galactose extracted from blood or dried blood spots on Guthrie cards obtained from control individuals and patients with a GALM deficiency. No significant differences in the galactose anomer profiles were observed between the controls and the patients (data not shown), probably because anomerization from one galactose anomer to the other occurs spontaneously and rapidly in water, ${ }^{30}$ resulting in a complete equilibrium between $\alpha$ - and $\beta$-D-galactose (Supplementary Figure S2). Further studies are necessary to establish chemical methods for diagnosing GALM deficiency.

In summary, to the best of our knowledge, this study is the first to report an association between biallelic pathogenic variants in GALM and a novel type of galactosemia (type IV galactosemia). We recommend that an analysis of GALM variants should be added to the diagnostic workup of patients with unexplained galactosemia.

\section{ELECTRONIC SUPPLEMENTARY MATERIAL}

The online version of this article (https://doi.org/10.1038/s41436$018-0340-x)$ contains supplementary material, which is available to authorized users.

\section{ACKNOWLEDGEMENTS}

The authors would like to thank the patients and families who participated in this study. We thank Yoko Chiba, Kumi Ito, Miyuki Tsuda, Mami Kikuchi, Makiko Nakagawa, Yoko Tateda and Kiyotaka Kuroda for providing technical assistance. We also acknowledge the support from the Biomedical Research Core of the Tohoku University Graduate School of Medicine and the Biomedical Research Unit of Tohoku University Hospital. This research was supported by the Japanese Agency for Medical Research and Development (AMED) through grant numbers JP17ek0109151h0003 (Initiative on Rare and Undiagnosed Diseases [IRUD] to S. Kure), JP17ek0109276 (to T.F.), and JP17km0405001 (the Program for Promotion of Genome Medicine to S. Koshiba). This work was also supported by grants from the Project for Promoting Public Utilization of Advanced Research Infrastructure (MEXT) (to S. Koshiba) and the Takeda Science Foundation (to A.K.).

\section{DISCLOSURE}

The authors declare no conflicts of interest.

\section{REFERENCES}

1. Holden HM, Rayment I, Thoden JB. Structure and function of enzymes of the Leloir pathway for galactose metabolism. J Biol Chem. 2003;278:43885-43888.
2. Coelho Al, Rubio-Gozalbo ME, Vicente JB, Rivera I. Sweet and sour: an update on classic galactosemia. J Inherit Metab Dis. 2017;40:325-342.

3. Timson DJ. The molecular basis of galactosemia-past, present and future. Gene. 2016;589:133-141.

4. Pasquali M, Yu C, Coffee B. Laboratory diagnosis of galactosemia: a technical standard and guideline of the American College of Medical Genetics and Genomics (ACMG). Genet Med. 2018;20:3-11.

5. Ohura T, Kobayashi K, Tazawa Y, et al. Neonatal presentation of adultonset type II citrullinemia. Hum Genet. 2001;108:87-90.

6. Tazawa $Y$, Kobayashi K, Ohura $T$, et al. Infantile cholestatic jaundice associated with adult-onset type II citrullinemia. J Pediatr. 2001; 138:735-740.

7. Timson DJ, Reece RJ. Identification and characterisation of human aldose 1-epimerase. FEBS Lett. 2003;543:21-24.

8. Reichardt JK, Woo SL. Molecular basis of galactosemia: mutations and polymorphisms in the gene encoding human galactose-1-phosphate uridylyltransferase. Proc Natl Acad Sci USA. 1991;88:2633-2637.

9. Leslie ND, Immerman EB, Flach JE, Florez M, Fridovich-Keil JL, Elsas LJ. The human galactose-1-phosphate uridyltransferase gene. Genomics. 1992;14:474-480.

10. Stambolian $D, A i Y$, Sidjanin $D$, et al. Cloning of the galactokinase CDNA and identification of mutations in two families with cataracts. Nat Genet. 1995:10:307-312.

11. Quimby BB, Alano A, Almashanu S, DeSandro AM, Cowan TM, FridovichKeil JL. Characterization of two mutations associated with epimerasedeficiency galactosemia, by use of a yeast expression system for human UDP-galactose-4-epimerase. Am J Hum Genet. 1997; 61:590-598.

12. Alano A, Almashanu S, Chinsky JM, et al. Molecular characterization of a unique patient with epimerase-deficiency galactosaemia. J Inherit Metab Dis. 1998;21:341-350.

13. Maceratesi P, Daude N, Dallapiccola B, et al. Human UDP-galactose 4 epimerase (GALE) gene and identification of five missense mutations in patients with epimerase-deficiency galactosemia. Mol Genet Metab. 1998;63:26-30

14. Holton JB, Gillett MG, MacFaul R, Young R. Galactosaemia: a new severe variant due to uridine diphosphate galactose-4-epimerase deficiency. Arch Dis Child. 1981;56:885-887.

15. Wohlers TM, Christacos NC, Harreman MT, Fridovich-Keil JL. Identification and characterization of a mutation, in the human UDPgalactose-4-epimerase gene, associated with generalized epimerasedeficiency galactosemia. Am J Hum Genet. 1999;64:462-470.

16. Waggoner DD, Buist NR, Donnell GN. Long-term prognosis in galactosaemia: results of a survey of 350 cases. J Inherit Metab Dis. 1990;13:802-818

17. Mizoguchi N, Ono H, Eguchi T, Sakura N. Galactose metabolites in blood from neonates with and without hypergalactosaemia detected by mass screening. Eur J Pediatr. 2000;159:851-853.

18. DePristo MA, Banks E, Poplin R, et al. A framework for variation discovery and genotyping using next-generation DNA sequencing data. Nat Genet. 2011:43:491-498.

19. Wang K, Li M, Hakonarson H. ANNOVAR: functional annotation of genetic variants from high-throughput sequencing data. Nucleic Acids Res. 2010;38:e164.

20. Higasa K, Miyake N, Yoshimura J, et al. Human genetic variation database, a reference database of genetic variations in the Japanese population. J Hum Genet. 2016;61:547-553.

21. Narahara M, Higasa K, Nakamura S. et al. Large-scale East-Asian eQTL mapping reveals novel candidate genes for LD mapping and the genomic landscape of transcriptional effects of sequence variants. PLOS ONE. 2014;9:e100924

22. Otsuki T, Ota T, Nishikawa T, et al. Signal sequence and keyword trap in silico for selection of full-length human cDNAs encoding secretion or membrane proteins from oligo-capped cDNA libraries. DNA Res. 2005;12:117-126.

23. Kimura K, Wakamatsu A, Suzuki Y, et al. Diversification of transcriptional modulation: large-scale identification and characterization of putative alternative promoters of human genes. Genome Res. 2006;16:55-65.

24. Ota T, Suzuki $Y$, Nishikawa $T$, et al. Complete sequencing and characterization of 21,243 full-length human cDNAs. Nat Genet. 2004;36:40-45.

25. Itoh $\mathrm{M}$, Yasunishi $\mathrm{A}$, Imamura $\mathrm{K}$, et al. Constructing ORFeome resources with removable termination codons. Biotechniques. 2006;41:44-46. 48passim 
26. Hui-Yuen J, McAllister $S$, Koganti $S$, Hill $E$, Bhaduri-Mclntosh $S$ Establishment of Epstein-Barr virus growth-transformed lymphoblastoid cell lines. J Vis Exp. 2011;57:e3321.

27. Beebe JA, Frey PA. Galactose mutarotase: purification, characterization, and investigations of two important histidine residues. Biochemistry. 1998;37:14989-14997.

28. Okuda J, Kawamura M. Rapid microdetermination of D-galactose anomers by $\beta$-D-galactose dehydrogenase and mutarotase. Jpn J Clin Chem. 1981;10:77-80.

29. Thoden JB, Timson DJ, Reece RJ, Holden HM. Molecular structure of human galactose mutarotase. J Biol Chem. 2004;279:23431-23437.

30. Levy GB, Cook ES. A rotographic study of mutarotase. Biochem J. 1954;57:50-55.

31. Fukasawa T, Sakurai $H$, Nogi $Y$, Baruffini E. Galactose transporters discriminate steric anomers at the cell surface in yeast. FEMS Yeast Res. 2009;9:723-731.

32. Bouffard GG, Rudd KE, Adhya SL. Dependence of lactose metabolism upon mutarotase encoded in the gal operon in Escherichia coli. J Mol Biol. 1994;244:269-278.
33. Bosch AM, Bakker $H D$, van Gennip $A H$, van Kempen JV, Wanders RJA, Wijburg FA. Clinical features of galactokinase deficiency: a review of the literature. J Inherit Metab Dis. 2002; 25:629-634.

34. Hennermann JB, Schadewaldt P, Vetter B, Shin YS, Mönch E, Klein J. Features and outcome of galactokinase deficiency in children diagnosed by newborn screening. J Inherit Metab Dis. 2011;34:399-407.

35. Openo KK, Schulz JM, Vargas CA, et al. Epimerase-deficiency galactosemia is not a binary condition. Am J Hum Genet. 2006;78:89-102.

36. Liu X, Cannon DM, Akula N, et al. A non-synonymous polymorphism in galactose mutarotase (GALM) is associated with serotonin transporter binding potential in the human thalamus: results of a genome-wide association study. Mol Psychiatry. 2011; 16:584-585.

37. Ng WG, Donnell GN, Bergren WR. Galactokinase activity in human erythrocytes of individuals at different ages. J Lab Clin Med. 1965;66:115-121. 